\title{
Investigation of Borrelia burgdorferi genotypes in Australia obtained from erythema migrans tissue
}

\section{Peter J Mayne}

International Lyme and Associated Diseases Society, Bethesda, MD, USA

The author is a member of the International Lyme and Associated Diseases Society (ILADS)
Correspondence: Peter J Mayne

89 Bold St, Laurieton,

NSW 2443, Australia

Tel +6I 265599277

Fax +6I 265597344

Email laurietonmedical@gmail.com
This article was published in the following Dove Press journal:

Clinical, Cosmetic and Investigational Dermatology

4 July 2012

Number of times this article has been viewed

Background: Lyme disease (LD) is an emerging infectious disease in Australia. There has been controversy regarding endemic lyme disease in the country for over 20 years. Borrelia burgdorferi sensu stricto (Bbss) and sensu lato $(B b s l)$ are closely related spirochetal species that are the causative agents of LD in humans. Clinical transmission of this tick-borne disease is marked by a characteristic rash known as erythema migrans (EM). This study employed molecular techniques to demonstrate the spirochetal agent of Lyme disease isolated from EM biopsies of patients in Australia and then investigate their genetic diversity.

Methods: Four patients who presented to the author's practice over a one-year period from mid 2010 to mid 2011 returned positive results on central tissue biopsy of EM lesions using polymerase chain reaction (PCR) analysis. The findings were confirmed by DNA sequencing, and basic local alignment search tool (BLAST) analysis was then used to genetically characterize the causative organisms.

Results: Three isolates were identified as Bbss that lay genotypically between strains B31 and ZS7 and were then characterized as strain $64 \mathrm{~b}$. One of the three isolates though may have similarity to $B$. bissettii a $B b s$. The fourth isolate was more appropriately placed in the sensu lato group and appeared to be similar, but not identical to, a B. valaisiana-type isolate. In this study, a central biopsy taken within 6 days of infection was used instead of conventional sampling at the leading edge, and the merits of this are discussed.

Conclusion: These patients acquired infection in Australia, further proving endemic LD on the continent. Central biopsy site of EM is a useful tool for PCR evaluation. BLAST searches suggest a genetic diversity of $B$. burgdorferi, which has implications concerning the diagnosis, clinical severity, and testing of LD in Australia.

Keywords: tissue biopsy, PCR, lyme disease, lyme-like

\section{Introduction}

Lyme disease (LD) is an increasing health burden on the Australian community requiring wider diagnostic recognition. ${ }^{1}$ Borrelia burgdorferi sensu lato (Bbsl) are closely related spirochetal species that are the causative agents of LD in humans. ${ }^{2}$ The author has previously reported the endemic presence of LD in Australia by positive serological and molecular testing of blood samples taken from symptomatic patients who have never been abroad, supporting the preliminary observations of others. ${ }^{1,3,4}$ In the current study, the presence of LD in Australia is confirmed by the detection of Borrelia species (spp.) using polymerase chain reaction (PCR) analysis of biopsy tissue taken from patients with a clinical presentation of erythema migrans (EM). The findings were confirmed by DNA sequencing. The use of central biopsy to replace leading-edge 
biopsy is explored, with indications it is more reliable in detecting Borrelia spp.. Positive findings led to analysis of the sequences deducing preliminary information on Australian genotypes consistent with both $B$. burgdorferi sensu stricto (Bbss) and $B b s l$, an important factor in accurate future identification and diagnosis of native infection.

\section{Materials and methods}

Skin punch biopsies were taken from the bite sites of four patients presenting with EM, each at a time interval between 1 and 6 days from bite to biopsy. Two biopsies were taken from patient $\mathrm{A}$, one from the central bite site and the other from the leading edge of the lesion. See Figure 1 at day 3 , the day of biopsy and commencement of treatment, and Figure 2 at day 6. The sample from the central biopsy of patient A was positive and the leading-edge sample was negative. Prior to this, the author had always been sampling from the leading edge of EM lesions, with consistently negative results. The result on patient $\mathrm{A}$ encouraged subsequent sampling using central biopsy only on $\mathrm{B}, \mathrm{C}$, and $\mathrm{D}$, which yielded the results in this study. Case $\mathrm{A}$ is the index case on a timeline of 1 year in this series. It is of note that all prior leading-edge biopsies taken before the day of patient A's presentation were analyzed with the same primers about to be discussed for patients $\mathrm{A}, \mathrm{B}$, and $\mathrm{C}$. The patients will be referred to as patients $\mathrm{A}, \mathrm{B}, \mathrm{C}$, and $\mathrm{D}$ in this discussion, and biopsies taken from them referred to as biopsies A, B, C, and $\mathrm{D}$ respectively.

All specimens were forwarded to Australian Biologics in Sydney for PCR analysis. For specimens A, B, and C, primers for borrelial RNA polymerase gene (rpoC) were employed for amplification using a set of one forward and one reverse. The sequences used are proprietary to Australian Biologics. Specimen D was analyzed with a multiplex set (see Table 1). It was analyzed differently as part of a process of attempting

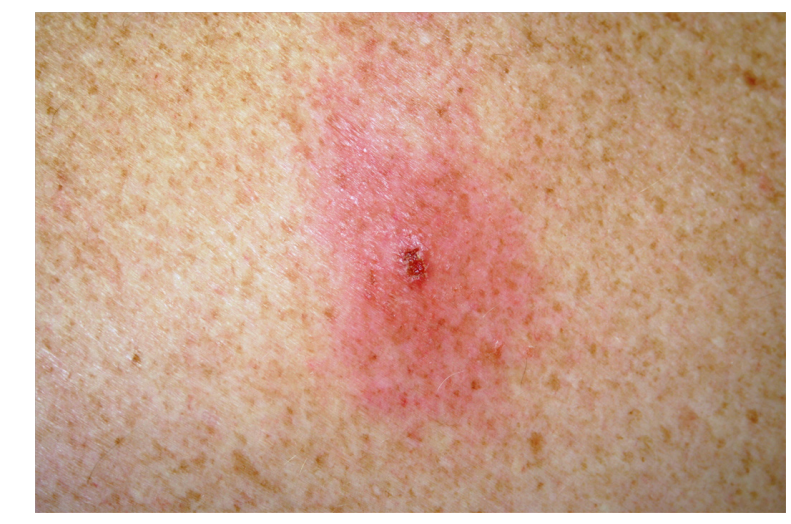

Figure I Erythema migrans patient A at day 3 (biopsy day).

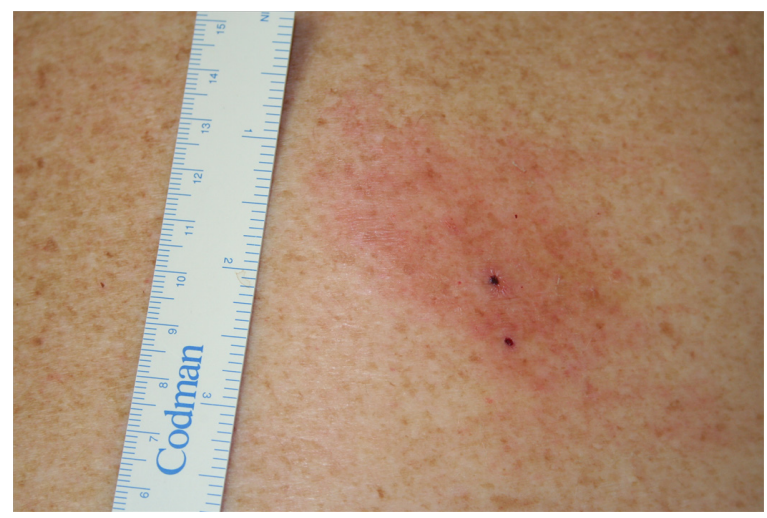

Figure 2 Erythema migrans patient $A$ at day 6.

to improve borrelial detection by that laboratory. Cycles, annealing temperatures, and amplicon size are available on request.

Positive Borrelia DNA product was then submitted to Australian Genome Research Facility (AGRF) in Sydney for confirmatory testing. AGRF is a National Association of Testing Authorities (NATA) accredited laboratory and is Australia's largest provider of genomics services and solutions. ${ }^{5}$ All four sequence results from AGRF were then submitted for basic local alignment search tool (BLAST) inquiry. ${ }^{6}$ The technique follows in the Discussion section. Then under alignments (third grouping using BLAST) tree analysis using neighbor joining was performed on the results. ${ }^{6}$ There were unnamed leaves. PathoSystems Resource Integration Center (PATRIC) was then used to examine the sequences, revealing additional findings. ${ }^{7}$ PATRIC was also used to construct a cladogram of all known Borrelia spp. (see Figure 3) but not from the study's data.

\section{Results}

The study identified DNA product suggestive of Borrelia spp. in all four samples. The sequences obtained on the respective specimens are presented in Table 2. Each sequence was submitted to BLAST inquiry using that sequence set. Clinicians may like to do the BLAST themselves. The author selected the following BLAST

Table I Multiplex primer set

\begin{tabular}{lll}
\hline & $\begin{array}{l}\text { Wills (University of } \\
\text { Newcastle ) primers } \\
\text { for I6S rRNA gene }\end{array}$ & $\begin{array}{l}\text { Crowder (et al 20 I 0) } \\
\text { Osp C gene }\end{array}$ \\
\hline Forward & 5' CCC TCA CTA AAC & TGA CGG TAT TTT TA TTT \\
& ATA CCT 3' & ATA TCT TGT AAT AAT \\
& & TGA GG 3' \\
Reverse & 5' ATC TGT TAC CAG & 5' TTT GCT TAT TTC TGT \\
& CAT GTA AT 3' & AAG ATT AGG CCC TTT 3' \\
\hline
\end{tabular}




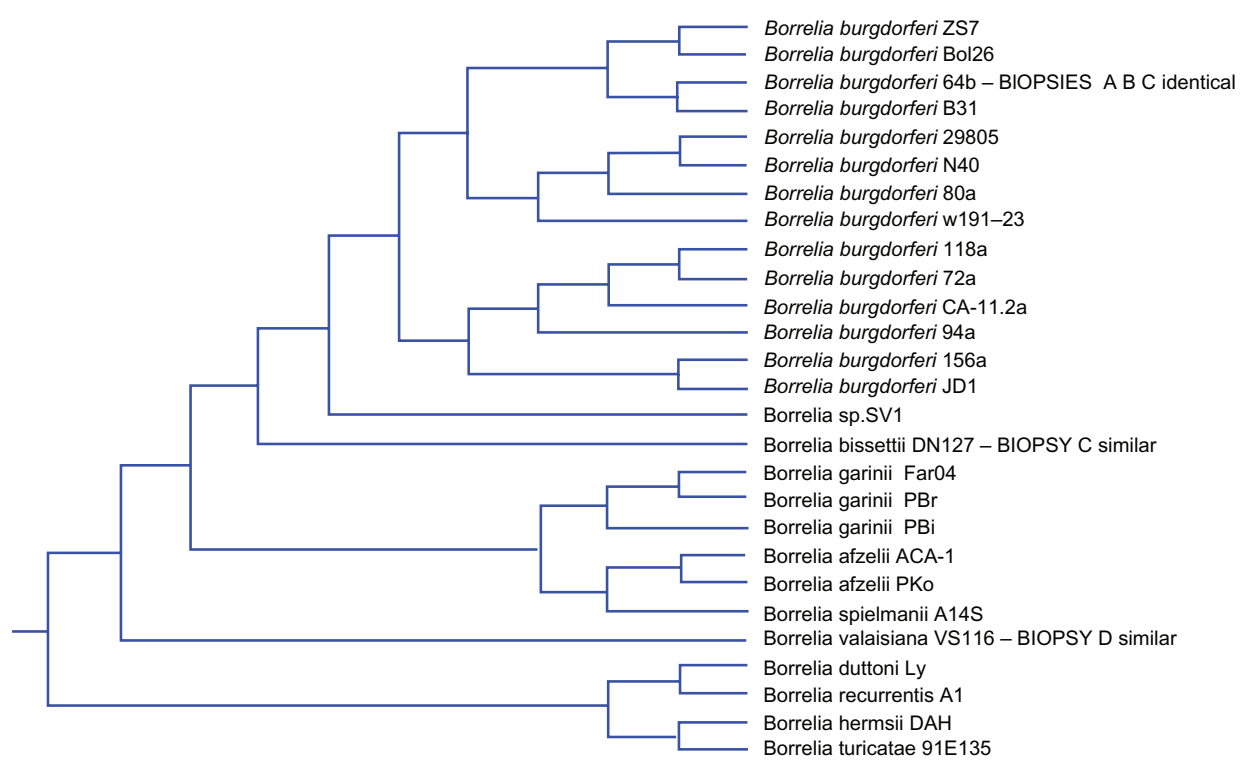

Figure 3 Cladogram of Borrelia species. Source PATRIC as discussed in text. Postion of BLAST findings annotated for Biopsies A-D.

Note: This cladogram was not produced from biopsy material.

parameters: nucleotide blast $>$ copy the biopsy's sequence into the "enter accession number" box $>$ database set and choose "others" > program selection and choose Megablast for highly similar sequences $>$ BLAST button at the bottom of the page. Results on A, B and C show detection of Borrelia DNA consistent with $B b$ ss (see Figure 3). In particular, results demonstrate strains N40, ZS7, and B31 as equal top alignments with the best match. Patient $\mathrm{B}$ has an equal ranking of JD1. This group of results shows a maximum identification (max ident) percentage between 97 and 99 and an extremely low expected value (E). Medical science places much emphasis on probability $(\mathrm{P})$ when interpreting results. Examining $P$ in the BLAST output above, the negative sign in the $\mathrm{E}$ value is a negative power. The mathematic constant "e" is 2.7 to one decimal place, and e is raised to that negative power. The distribution of a sequence analysis of this type is a Poisson distribution. ${ }^{8}$ It is currently believed that with high letter count submissions in these types of sequences that the $\mathrm{E}$ value is thought to approximate probability. ${ }^{8}$ The $E$ value is the expected number of high scoring pairs for a certain nominated score when comparing the alignment of local pairs. ${ }^{8}$ Algorithms find all segment pairs whose scores cannot be improved by extension or trimming. ${ }^{8}$

Analysis of the alignments using neighbor joining shows patient $\mathrm{A}$ and $\mathrm{B}$ samples to be lying identically between $B b$ ZS7 and $B b$ B31 (see Figure 4). ${ }^{6}$ Two unnamed nodes lay between those results which are further analyzed below using PATRIC. For patient $\mathrm{C}$, however, the picture is very

Table 2 DNA sequences

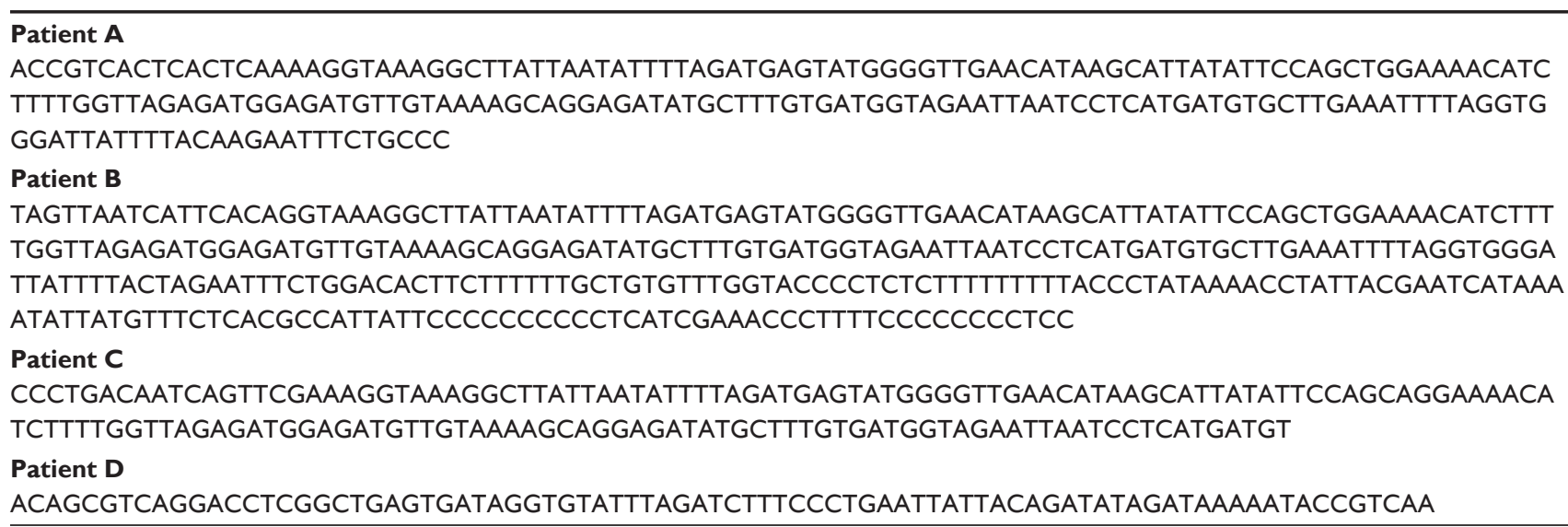


Table 3 Top scoring sequences

\begin{tabular}{|c|c|c|c|c|c|c|}
\hline Accession & Description & Max score & Total score & Query coverage & E value & Max ident \\
\hline \multicolumn{7}{|l|}{ Patient A } \\
\hline CP002228.I & Borrelia burgdorferi N40, complete genome & 339 & 339 & $91 \%$ & $6.00 \mathrm{E}-90$ & $99 \%$ \\
\hline CP00I205.I & Borrelia burgdorferi ZS7, complete genome & 339 & 339 & $91 \%$ & $6.00 \mathrm{E}-90$ & $99 \%$ \\
\hline AE000783.I & Borrelia burgdorferi B3I, complete genome & 339 & 339 & $91 \%$ & $6.00 \mathrm{E}-90$ & $99 \%$ \\
\hline CP0023I2.I & Borrelia burgdorferi JDI, complete genome & 333 & 333 & $91 \%$ & $3.00 \mathrm{E}-88$ & $98 \%$ \\
\hline \multicolumn{7}{|l|}{ Patient B } \\
\hline CP002228.I & Borrelia burgdorferi N40, complete genome & 327 & 327 & $55 \%$ & $2.00 \mathrm{E}-86$ & $98 \%$ \\
\hline CP00I205.I & Borrelia burgdorferi ZS7, complete genome & 327 & 327 & $55 \%$ & $2.00 \mathrm{E}-86$ & $98 \%$ \\
\hline AE000783.I & Borrelia burgdorferi B3I, complete genome & 327 & 327 & $55 \%$ & $2.00 \mathrm{E}-86$ & $98 \%$ \\
\hline CP0023 I 2.I & Borrelia burgdorferi JDI, complete genome & 322 & 322 & $55 \%$ & $\mathrm{I} .00 \mathrm{E}-84$ & $98 \%$ \\
\hline \multicolumn{7}{|l|}{ Patient C } \\
\hline CP002228.I & Borrelia burgdorferi N40, complete genome & 267 & 267 & $95 \%$ & $2.00 \mathrm{E}-68$ & $97 \%$ \\
\hline CP00I205.I & Borrelia burgdorferi ZS7, complete genome & 267 & 267 & $95 \%$ & $2.00 \mathrm{E}-68$ & $97 \%$ \\
\hline AE000783.I & Borrelia burgdorferi B3I, complete genome & 267 & 267 & $95 \%$ & $2.00 \mathrm{E}-68$ & $97 \%$ \\
\hline CР0023I2.I & Borrelia burgdorferi JDI, complete genome & 261 & 261 & $95 \%$ & I.00E-66 & $96 \%$ \\
\hline
\end{tabular}

different. The result on phylogenetic tree analysis appears in the neighborhood of, but distinct from, bissettii DN127, in spite of initial indications suggesting similarity to A and B (see Figure 5). ${ }^{6}$ BLAST comparison alignment using "bl2seq" (this is a selection on the BLAST site for aligning any two sequences) demonstrated that samples A and B are identical (see Table 4). A further paired alignment between samples A and $\mathrm{C}$ shows it is very similar in equality but not quite as strong (see Table 5). Both sets of paired alignments had no gaps. Results for BLAST analysis of cloned product from patient $\mathrm{D}$ returns "No significant similarity found". Then using blastn (comparison for similar sequences) instead of choosing Megablast for highly similar sequences, a $B$. valaisiana-like species is demonstrated. ${ }^{6}$ Attempts with "bl2seq" to align A, $\mathrm{B}$, and $\mathrm{C}$ to $\mathrm{D}$ fail. A cladogram of the entire borrelial tree is presented in Figure 3. It was produced using PATRIC by choosing organisms $>$ bacteria $>$ phylogeny then selecting order spirochaetales and cladogram. ${ }^{7}$ The result was trimmed to Borrelia spp. The findings from NIHC BLAST were then superimposed in Figure 3 to demonstrate their position. $\mathrm{C}$ is duplicated.

The sequence data was then analyzed at PATRIC producing rather different results. ${ }^{7}$ For each specimen, the output is some 1400 lines in a spreadsheet. Results are summarized here and show that $\mathrm{A}, \mathrm{B}$, and $\mathrm{C}$ had identical matches with max ident of $99 \%$ and $\mathrm{E}$ value of $9 \times 10^{-95}$ to

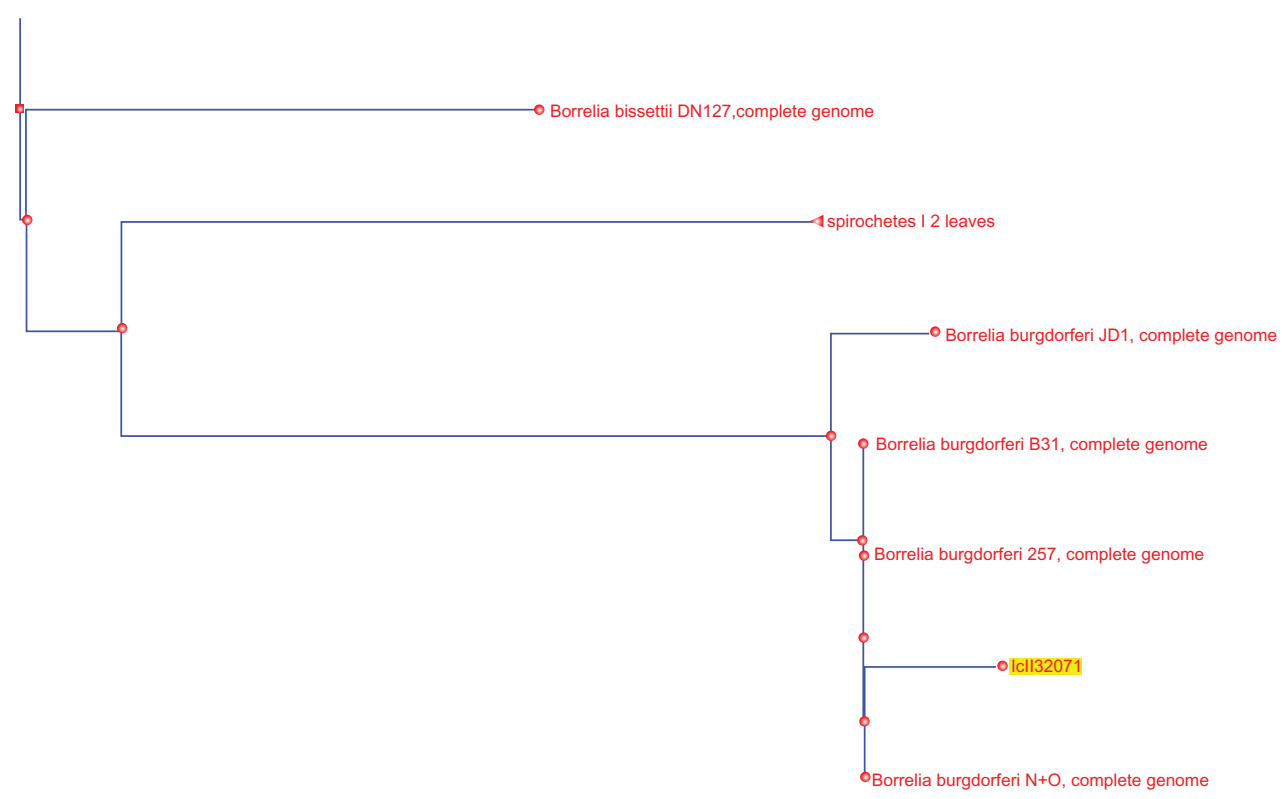

Figure 4 Distance tree of results $A$ and $B$ using neighbor joining construction. 


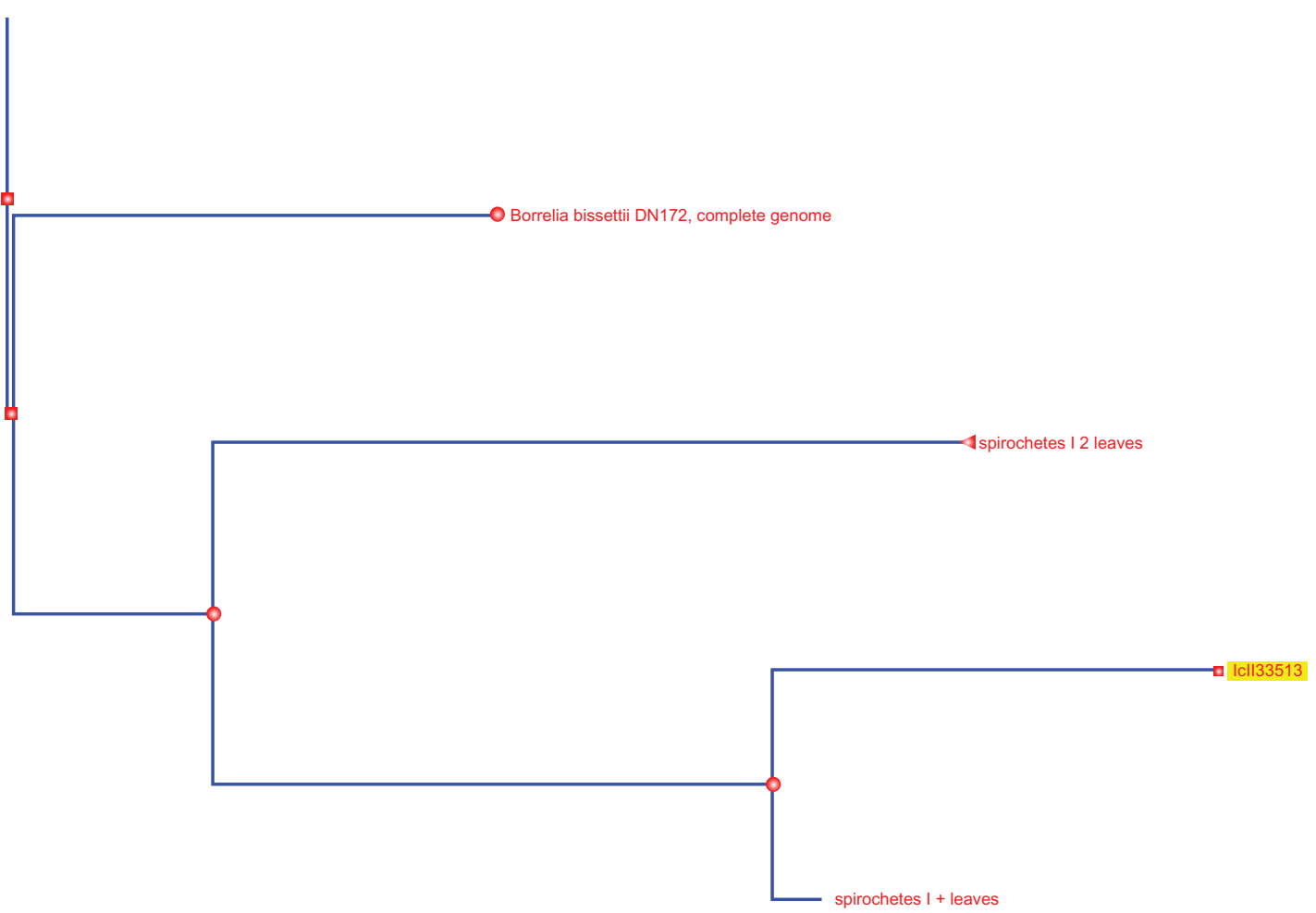

Figure 5 Distance tree of results $C$ using neighbor joining construction.

Table 4 Paired alignment patients A B

Blast 2 sequences

Nucleotide sequence (336 letters)

Sequences producing significant alignments

\begin{tabular}{|c|c|c|c|c|c|c|c|}
\hline Accession & Description & Max score & Total score & Query coverage & E value & Max ident & Links \\
\hline 59891 & & 339 & 339 & $55 \%$ & $5.00 \mathrm{E}-98$ & $99 \%$ & \\
\hline
\end{tabular}

Alignments

$>|c||5989|$

Length $=206$

Score $=339$ bits (183), Expect $=5 e-98$

Identities $=$ I85/I86 (99\%), Gaps $=0 / 186(0 \%)$

Strand $=$ Plus/Plus

Query 16 AGGTAAAGGCTTATTAATATTTTAGATGAGTATGGGGTTGAACATAAGCATTATATTCCA 75 |||||||||||||||||||||||||||||||||||||||||||||||||||||||||||||

Sbjct 18 AGGTAAAGGCTTATTAATATTTTAGATGAGTATGGGGTTGAACATAAGCATTATATTCCA 77

Query 76 GCTGGAAAACATCTTTTGGTTAGAGATGGAGATGTTGTAAAAGCAGGAGATATGCTTTGT I 35

|||||||||||||||||||||||||||||||||||||||||||||||||||||||||| $\mid$

Sbjct 78 GCTGGAAAACATCTTTTGGTTAGAGATGGAGATGTTGTAAAAGCAGGAGATATGCTTTGT I 37

Query 136 GATGGTAGAATTAATCCTCATGATGTGCTTGAAATTTTAGGTGGGATTATTTTACTAGAA 195

|||||||||||||||||||||||||||||||||||||||||||||||||||||||||| ||||

Sbjct I 38 GATGGTAGAATTAATCCTCATGATGTGCTTGAAATTTTAGGTGGGATTATTTTACAAGAA I 97

Query 196 TTTCTG 20I

||||||

Sbjct 198 TTTCTG 203 
Table 5 Paired alignment patients A C

\begin{tabular}{|c|c|c|c|c|c|c|c|}
\hline $\begin{array}{l}\text { Blast } 2 \text { se } \\
\text { Nucleotid } \\
\text { Sequences }\end{array}$ & $\begin{array}{l}\text { ces } \\
\text { quence ( } 165 \\
\text { ucing significar }\end{array}$ & $\begin{array}{l}\text { ns) } \\
\text { nments }\end{array}$ & & & & & \\
\hline Accession & Description & Max score & Total score & Query coverage & E value & Max ident & Links \\
\hline 55793 & & 268 & 268 & $89 \%$ & 4.00E-77 & $99 \%$ & \\
\hline \multicolumn{8}{|l|}{ Alignments } \\
\hline \multicolumn{8}{|l|}{$>|c| \mid 55793$} \\
\hline \multicolumn{8}{|l|}{ Length $=206$} \\
\hline \multicolumn{8}{|c|}{ Score $=268$ bits $(145)$, Expect $=4 \mathrm{e}-77$} \\
\hline \multicolumn{8}{|c|}{ Identities $=\mid 47 / / 48(99 \%)$, Gaps $=0 / / 48(0 \%)$} \\
\hline \multicolumn{8}{|c|}{ Strand $=$ Plus/Plus } \\
\hline \multicolumn{8}{|c|}{$\begin{array}{l}\text { Query I8 AAAGGTAAAGGCTTATTAATATTTTAGATGAGTATGGGGTTGAACATAAGCATTATATTC } 77 \\
\text {. }\end{array}$} \\
\hline \multicolumn{8}{|c|}{ Sbjct I6 AAAGGTAAAGGCTTATTAATATTTTAGATGAGTATGGGGTTGAACATAAGCATTATATTC 75} \\
\hline \multicolumn{8}{|c|}{ Query 78 CAGCAGGAAAACATCTTTTGGTTAGAGATGGAGATGTTGTAAAAGCAGGAGATATGCTTT I37 } \\
\hline \multicolumn{5}{|c|}{||||||||||||||||||||||||||||||||||||||||||||||||||||||||||||} & & & \\
\hline \multicolumn{8}{|c|}{ Query I38 GTGATGGTAGAATTAATCCTCATGATGT I65 } \\
\hline $\begin{aligned} \||||||| \\
\text { Sbjct I } 36 \mathrm{GT}\end{aligned}$ & $\begin{array}{l}\|\|\|\||||| \mid \\
\text { GGTAGAATT }\end{array}$ & CTCATGATC & & & & & \\
\hline
\end{tabular}

all of the following Borrelia spp. equally: ZS7, W191-23, N40, CA-11.2A, Bol126, B31, 94a, 72a, 64b, 29805, 156a, and 118a. After several more Borrelia genotypes are listed at much lower max ident and E values, one finds the first non-borrelial bacterium is fusobacterium with an $\mathrm{E}$ of just 0.029 , and then listeria and campylobacter. This is very compelling evidence of Borrelia spp. Specimen D had a much lower confidence band, with six of the above genotypes at an $\mathrm{E}$ of $2 \times 10^{-4}$. The first non-borrelial listings were three clostridium species with an E of 0.16. Specimen D may be a novel genotype.

BLAST comparison alignment using "bl2seq" from NCBI of the sequences was then used in an attempt to further analyze the specimens using deposited sequences for known Borrelia spp. from Genomic Sequencing Center for Infectious Diseases, University of Maryland, USA, this time demonstrating the $64 \mathrm{~b}$ serotype. ${ }^{6}$ The accession number used for whole genome shotgun sequencing and resulting match was $\mathrm{Bb}$ 64b ABKA02000001.1. Biopsies $\mathrm{A}, \mathrm{B}$, and $\mathrm{C}$ were all a positive match. No match could be found for D.

\section{Discussion}

Borrelia-specific DNA was detected in all four patient samples. The author proposes detection of Borrelia is more likely to be successful when the specimen is taken from the central bite site than when taken from the leading edge in early lesions. This contradicts convention, the standard long-held practice of biopsy from the leading edge for which there is no published data on PubMed searching on February 10, 2012 using the headings erythema migrans tissue biopsy. Adding the term peripheral finds a paper examining central versus peripheral biopsy by Jurca et al, where the authors conclude that detection from peripheral and central sites was equivalent. ${ }^{9}$ It has been thought that Borrelia DNA detection from EM rashes at the leading edge is successful in $80 \%$ of cases. ${ }^{10}$ This statement by Sydney University is not itself referenced. The current study suggests that employing central site of biopsy may reliably provide detectable spirochetal DNA evidence up to 6 days after tick attachment, even after tick removal. All patients received their tick bite within 10 kilometers of the eastern coastline in the state of New South Wales. A and B were only $12 \mathrm{~km}$ apart but with a time gap of 1 year. Patient $\mathrm{C}$ was in the northern suburbs of Sydney. Patient D was some $80 \mathrm{~km}$ north of patients A and B. Patient D had presented multiple times with EM over a 1-year period. He had protracted chronic LD, and lesion edge biopsies were negative prior to this attempt to determine whether a B. burgdorferi spp. was responsible. On this last presentation, a biopsy taken from the central bite 
site was positive for B. burgdorferi spp., again supporting the concept of central biopsy.

For the past two decades there has been considerable debate concerning the existence of LD on the continent of Australia. There are two longstanding published reports of both EM and locally acquired human $B$. burgdorferi infection and one contrary. ${ }^{3,411}$ At the time of the McCrossin study in 1986, only serological diagnostic tests were available. ${ }^{3}$ So sequencing was not possible for confirmation of those results. The Russell et al study in 1994 examined the midgut content of over 12,000 mostly unfed ticks with no spirochetes found. ${ }^{11}$ However, it is now known that spirochetes within flat, or unfed, nymphs exist in low numbers and in a poorly understood metabolic state that enables them to endure prolonged periods of nutrient deprivation. In this state, the transcription regulators Rrp2-RpoN-RpoS and the hybrid histidine Hk1-Rrp1 pathways are inactive, as are mammalian-phase genes, while tick-phase genes are maximally expressed. ${ }^{2}$ At the commencement of feeding, this status is reversed with rapid replication. ${ }^{2}$ Then, under the heading "Within the mammalian host" a clear model of salivary hypostome spirochete movement from tick to skin is described. ${ }^{2}$ The Russell study's negative findings may no longer be appropriate. The Hudson study in 1998 described a cultured isolate of Borrelia identified by molecular testing from one patient who had travelled overseas but due to the length of time since overseas travel ( 17 months) it was believed the patient acquired their infection in Australia. Sequencing results suggest similarity to a European strain of $B$. garinii, rather than the $B$. garinii species typically described in Asia. ${ }^{4}$ In a 2011 study, the current author published serological and molecular evidence of endemic Lyme borreliosis in Australia, reporting positive $\mathrm{LD}$ test results of whole blood and serum taken from patients who had never left the country. ${ }^{1}$ The current study provides further proof of endemicity of Borrelia infection in Australian patients using molecular detection of Borrelia DNA from biopsy material. This is a small study based on four samples. Further investigation is required to determine if there are novel Borrelia genotypes in that a $B$. bisettii-like organism and a $B$. valaisiana-like organism are suggested from the data of patients $C$ and $D$ respectively. Further genotyping studies should be done to confirm or disprove the serotype $64 \mathrm{~b}$ nature of specimens $\mathrm{A}$ and $\mathrm{B}$.

In the expanding universe of B. burgdorferi, in addition to small mammals, transhemispheric bird migration is responsible for spirochete dissemination. ${ }^{2,12}$ Although Australia has approximately 75 tick species, it is generally accepted that Ixodes spp., and Ixodes holocyclus (Ih) in particular, are the main contenders in transmitting human tick-borne diseases. ${ }^{13,14}$ The Eastern coastline is habitat to Ih, which is known to vector human disease, including rickettsial infection and tick paralysis. ${ }^{1,13-16}$ The Ih tick is colloquially named paralysis tick. ${ }^{10}$ It may also be called grass tick, shell back tick, and several others. It thrives in humid coastal conditions, mainly in flatlands, from the north of the continent just above Cooktown in Queensland to Lakes Entrance in Victoria at the very south of the continent. ${ }^{10}$ This region contains a very high proportion of Australia's human population.

The Ih tick has larval, nymph, and adult stages all of which require a blood meal. ${ }^{10}$ Larvae typically feed upon small animal hosts, whilst the nymph and adult will also feed upon larger animals. ${ }^{2,10}$ Humans are incidental hosts to all three forms, but the nymph is primarily responsible for $90 \%$ of human tick-borne disease. ${ }^{2}$ The tick may stay attached for up to 5-6 days before detaching if not found. Many tick bites are not observed or reported. ${ }^{1}$ In Australia, the majority of bites will come to nothing more than an erythematous macule (nonelevated) on the skin, of up to $2 \mathrm{~cm}$ diameter, resolving completely over a few days, providing the tick is removed promptly. Some lesions grow at the bite site to form a $2-3 \mathrm{~cm}$ papule by the second day, showing a black central eschar. ${ }^{17}$ Such a presentation at the bite site is the typical appearance of Queensland tick typhus, a rickettsial infection, and if untreated, this can be followed by a "spotted rash" of widespread distribution known as rickettsial spotted fever, a febrile illness whose erythematous macules are typically 4-5 mm in diameter. ${ }^{15,16}$

EM is a local clinical manifestation of $B b s$ infection. ${ }^{2,18,19}$ The incubation period is $3-32$ days. ${ }^{2,20}$ At this stage, the term early LD is also used, and this terminology includes early systemic manifestations such as meningitis, cranial nerve neuropathy, or carditis. An EM documented on an individual after being in a known endemic area is considered sufficient for a clinical diagnosis of LD; however, the single primary lesion must reach more than $5 \mathrm{~cm}$ in size to be classified as diagnostic and to be considered pathognomonic. ${ }^{21}$ The EM is often described as an initial $1 \mathrm{~cm}$ macular erythematous lesion, that at day 2-5 or even later, grows in diameter and thickens, lasting for up to 14 days or more if left untreated. Spirochetes are deposited into the skin during initial attachment. ${ }^{2}$ In the Northern Hemisphere, infection is rare in the first 24 hours after tick bite but most likely after 48 hours. ${ }^{2}$ Australia does not have enough data to confirm whether this is true locally.

LD is a protean multisystem illness that develops after a tick bite transmitting human pathogenic Borrelia 
spirochete when the initial local reaction is not treated or is under treated. ${ }^{22}$ Nomenclature of the disease includes the use of the terminology early disseminated LD and late disseminated LD. ${ }^{23}$ The International Lyme and Associated Diseases Society uses the terminology chronic LD for infection lasting more than 12 months. ${ }^{22}$ In North America, a principal symptom of the disease is an arthritic illness that may cause severe pain and swelling, especially in large joints, and can be associated with marked general fatigue and other somatic features. ${ }^{22}$ The Centers for Disease Control and Prevention website states that in the musculoskeletal system, LD produces recurrent attacks of arthritis with objective joint swelling in one or a few joints, sometimes followed by chronic arthritis. ${ }^{23}$ LD in North America may also be present as a neurological disease, which is generally accepted to be the principal manifestation throughout Europe and Asia. ${ }^{24}$ The author has presented similar findings of neurological manifestation with locally acquired LD in Australia with no arthropathy. ${ }^{1}$
Current thought is that Borrelia spp. are found along the eastern coastline of Australia and transmitted to humans by Ih. ${ }^{1}$ This study is implicating the same vector for transmission. Patients A and B received their tick at the same latitude but with a time gap of 1 year. Clinically, both patients reported painful itchy swellings of their EM. From a clinical standpoint, the two $B b$ ss genotypes caused an infection characterized by an intensely painful itchy eruption, one without significant systemic event. Refer to Table 6 for a summary of the clinical detail. On the contrary, in patient $\mathrm{C}$, the EM eruption was not particularly sore or itchy. This infection, with the possible $B$. bissettii-like genotype, in addition to producing local reaction, produced a profound systemic meningitic illness that lasted many weeks. The infection was contracted in the northern beachside region of Sydney.

There is conflict in the findings for patient $\mathrm{C}$ with one method of analysis, suggesting $B b s s 64 \mathrm{~b}$ and the other (neighbor joining tree) a similarity to $B$. bissettii. This distinction needs further research and clarification, and in particular,

Table 6 Demographic data and clinical details

\begin{tabular}{|c|c|c|c|c|}
\hline & \multicolumn{4}{|l|}{ Patient } \\
\hline & $\mathbf{A}$ & B & C & D \\
\hline Gender & Male & Female & Female & Male \\
\hline Overseas travel & Yes & Never & Yes & Never \\
\hline Geographic & $152.8 \mathrm{E}$ & I52.7E & $15 \mid .3 \mathrm{E}$ & $152.8 \mathrm{E}$ \\
\hline coordinates & $31.66 \mathrm{~S}$ & $31.73 \mathrm{~S}$ & $33.74 S$ & $31.32 S$ \\
\hline Systemic illness & No & Yes & $\begin{array}{l}\text { Very severe flu-like illness developed on } \\
5 \text { th day, including fever and meningism with severe } \\
\text { headache worse with coughing and shaking of } \\
\text { the head, photophobia and retro-orbital pain. } \\
\text { She developed no detectable neurological deficit }\end{array}$ & No \\
\hline Treatment & Doxycyline & Doxycyline & Amoxycillin plus clavulanate & Doxycyline \\
\hline Dose & 400 mg daily & 400 mg daily & $875 / 125 \mathrm{mg}$ bd & 400 mg daily \\
\hline Cautions & & & Breast feeding & \\
\hline Recovery factors & $\begin{array}{l}\text { Efficient } \\
\text { progress }\end{array}$ & $\begin{array}{l}\text { Supply interruption at } \\
\text { I week for I week and } \\
\text { developed flu-like symptoms. } \\
\text { Therapy resumed and } \\
\text { progress good }\end{array}$ & $\begin{array}{l}\text { Failed response. Eventually stopped breast feeding } \\
\text { at } 8 \text { weeks, and doxycycline } 400 \mathrm{mg} \text { daily added. } \\
\text { Within days, changed to clarithromycin because } \\
\text { of nausea. Response ensued }\end{array}$ & $\begin{array}{l}\text { Efficient } \\
\text { progress }\end{array}$ \\
\hline $\begin{array}{l}\text { Recovery time } \\
\text { Follow-up testing } \\
\text { at } 19 \text { weeks Igenex, } \\
\text { Palo Alto, USA }\end{array}$ & 4 weeks & 8 weeks & 12 weeks & 4 weeks \\
\hline $\begin{array}{l}\text { Borrelia burgdorferi } \\
\operatorname{lgA}, G \text {, and } M\end{array}$ & & & Negative & \\
\hline $\begin{array}{l}\text { B. burgdorferi } \\
\text { multiplex PCR }\end{array}$ & & & Negative & \\
\hline $\begin{array}{l}\text { Babesia microtii and } \\
\text { Babesia duncanii }\end{array}$ & & & Negative & \\
\hline Bartonella henselae & & & Negative & \\
\hline
\end{tabular}


note should be taken that clinical manifestation was different. Patient $\mathrm{C}$ received the tick bite in Northern Sydney. It is of clinical significance that this infection could not be controlled quickly with the amoxycillin clavulanate combination taken orally, and is an indicator of possible coinfection of babesiosis or bartonellosis. The author has already presented the independent incidence rate of these as $31 \%$ and $21 \%$ in Australia in associated tick-born diseases. ${ }^{1}$

Patient D had symptoms of protracted neurological LD on first presentation and had leading edge skin biopsies of EM done by the author, which returned negative as discussed above. Central biopsy on this occasion was positive and showed the $B$. valaisiana-like organism. This being the first human report of such a species in Australia warrants further research.

The terminology "Lyme-like" crops up frequently in Sydney, Australia. There are no published reports of a Lymelike illness in Australia using a PubMed search with the term "Lyme-like Australia" on January 24, 2012. The term "Lymelike illness" on this continent could now be considered, as the study presents some identity pointer to the wider Borrelia spp. infections as in patient D. "Lyme-like illness" has differing connotations. It is used appropriately to describe clinical infection by Borrelia that fall outside the sensu lato group, particularly in Europe and the USA where there are an increasingly growing number of genospecies of Borrelia being identified. It is being wrongly interpreted by media and Australian compensation insurance companies as a possible Lyme illness, lacking serological proof of borrelial infection. The identity of the genotype in patient $\mathrm{D}$ would appear to fit this appellation of Lyme-like illness.

Although the sample size is small, the relationship between the clinical presentations, the genotype involved, and geographical distribution demonstrated here may all be relevant to the development of local testing modalities, successful diagnosis of LD, and the subsequent clinical severity of disease.

\section{Conclusion}

Firstly, the results provide objective evidence confirming endemic LD in Australia by detecting and characterizing Borrelia genotypes of the Bbss group from biopsies of EM. Thus, LD must be considered to be a potential health risk in Australia. Possible identity to a Lyme-like illness in Australia is suggested as a valaisiana-like genotype.

Secondly, it is suggested that the success of Borrelia spp. detection from EM tissue can be greatly improved.
The author's findings indicate that during the first week of EM eruption, tissue biopsy at the bite site is preferable to that at the leading edge.

Thirdly, the evidence provided suggests that further research is needed to elucidate the genetic diversity of Borrelia spp. and B. burgdorferi genotypes causing LD on the continent of Australia. At least two and possibly three distinct genotypes are suggested, one characteristic of Bbss genetically lying between strains B31 and ZS7 and looking like $64 \mathrm{~b}$, one possibly related to, but discrete from, B. bissettii DN127, and finally a B. valaisiana-like genotype. This is a small study on four patients. More extensive studies are needed to make firmer conclusions. The genetic diversity of Borrelia spp. associated with LD has diagnostic laboratory and clinical significance to the medical profession and has implications for assessment of public health risk.

\section{Acknowledgments}

The author thanks Jennifer Burke MSc, Dr Peter Irwin, Dr Andrew Ladhams, Marianne Middleveen MSc, Janet Sperling MSc, Dr Felix Sperling, and Dr Raphael Stricker for their advice in the preparation of this paper.

\section{Disclosure}

The author reports no conflicts of interest in this work.

\section{References}

1. Mayne PJ. Emerging incidence of Lyme borreliosis, babesiosis, bartonellosis, and granulocytic ehrlichosis in Australia. Int J Gen Med. 2011;4:845-852.

2. Radolf J, Caimano M, Stevenson B, Hu L. Of ticks, mice and men: understanding the dual-host lifestyle of Lyme disease spirochaetes. Nat Rev Microbiol. 2012;10:87-99.

3. McCrossin I. LD on the NSW south coast. Med J Aust. 1986;144:724-725.

4. Hudson B, Stewart M, Lennox V, et al. Culture-positive Lyme borreliosis. Med J Aust. 1998;168:500-502.

5. AGRF Australian Genome Research Facility Ltd [homepage on the Internet]. Sydney, Australia: Westmead Millenium Institute. Available from: http://www.agrf.org.au. Accessed February 11, 2012.

6. BLAST ${ }^{\circledR}$ Basic Alignment Search Tool [homepage on the Internet]. Bethesda, MD: National Institutes of Health, National Center for Biotechnology Information. Available from: http://blast.ncbi.nlm.nih.gov. Accessed February 11, 2012.

7. PATRIC, Pathosystems Resource Integration Center. Bacteria spirocheates phylogenetic tree cladogram. Blacksburg, VA: Virginia Bioinformatics Institute. Available from: http://patricbrc.org/portal/portal/patric/ Phylogeny?cType=taxon\&cId=203691. Accessed February 11, 2012.

8. NCBI. The statistics of sequence similarity scores. Bethesda, MD: National Institutes of Health, National Center for Biotechnology Information. Available from: http://www.ncbi.nlm.nih.gov/BLAST/tutorial/ Altschul-1.html. Accessed February 11, 2012.

9. Jurca T, Ruzić-Sabljić E, Lotric-Furlan S, et al. Comparison of peripheral and central biopsy sites for the isolation of Borrelia burgdorferi sensu lato from erythema migrans skin lesions. Clin Infect Dis. 1998;27:636-638 
10. Department Medical Entomology. Lyme disease. Sydney, Australia: University of Sydney [updated November 7, 2003]. Available from: http://medent.usyd.edu.au/fact/lyme\%20disease.htm\#diag. Accessed February 11, 2012.

11. Russell RC, Doggett SL, Munro R, et al. Lyme disease: a search for the causative agent in south-eastern Australia. Epidemiol Infect. 1994;112(2):375-384

12. Olsen B, Duffy DC, Jaenson TG, Gylfe A, Bonnedahl J, Bergström S. Transhemispheric exchange of Lyme disease spirochetes by seabirds. J Clin Microbiol. 1995;33(12):3270-3274.

13. NSW Government Health. Ticks. NSW, Australia: NSW Government. Available from: http://www.health.nsw.gov.au/factsheets/general/ ticks_factsheet.html. Accessed February 11, 2012.

14. Murtagh J. Murtagh's General Practice. 5th ed. Sydney: McGraw-Hill Australia Pty Ltd; 2011:267.

15. Unsworth B, Stenos J, Graves S, et al. Flinders Island spotted fever rickettsioses caused by "marmionii” strain of Rickettsia honei, Eastern Australia. Emerg Infect Dis. 2007;13(4):566-573.

16. Unsworth N, Graves S, Nguyen C, Kemp G, Graham J, Stenos J. Markers of exposure to spotted fever rickettsiae in patients with chronic illness, including fatigue, in two Australian populations. QJM. 2008;101(4):269-274.

17. Department of Health. Infectious diseases, epidemiology and surveillance. Victoria, Australia: State Government of Victoria. Available from: http://ideas.health.vic.gov.au/bluebook/rickettsial.asp. Accessed February 11, 2012.
18. DermNet NZ. Lyme disease. Palmerston North, New Zealand: New Zealand Dermatological Society. Available from: http://www.dermnetnz. org/bacterial/lyme.html. Accessed February 11, 2012.

19. Müllegger RR, Glatz M. Skin manifestations of lyme borreliosis: diagnosis and management. Am J Clin Dermatol. 2008;9(6):355-368.

20. Radolf J, Salazar J, Dattwyler R. Borrelia: molecular biology, host interaction, and pathogenesis. Norfolk, UK: Caister Academic; 2010: 487-533.

21. Feder H, Abeles M, Bernstein M, Whitaker-Worth D, Grant-Kels J. Diagnosis, treatment, and prognosis of erythema migrans and Lyme arthritis. Clin Dermatol. 2006;24(6):509-520.

22. International Lyme and Associated Diseases Society. About Lyme disease. Bethesda MD: International Lyme and Associated Diseases Society. Available from: http://www.ilads.org/lyme_disease/about_lyme. html. Accessed February 11, 2012.

23. CDC. Lyme disease signs and symptoms. Atlanta, GA: Centers for Disease Control and Prevention. Available from: http://www.cdc.gov/ lyme/signs_symptoms. Accessed February 11, 2012.

24. Rudenko N, Golovchenko M, Grubhoffer L, Oliver JH Jr. Updates on Borrelia burgdorferi sensu lato complex with respect to public health. Ticks Tick Borne Dis. 2011:2(3):123-128.
Clinical, Cosmetic and Investigational Dermatology

\section{Publish your work in this journal}

Clinical, Cosmetic and Investigational Dermatology is an international, peer-reviewed, open access, online journal that focuses on the latest clinical and experimental research in all aspects of skin disease and cosmetic interventions. All areas of dermatology will be covered; contributions will be welcomed from all clinicians and

\section{Dovepress}

basic science researchers globally. This journal is indexed on CAS The manuscript management system is completely online and includes a very quick and fair peer-review system, which is all easy to use. Visit http://www.dovepress.com/testimonials.php to read real quotes from published authors. 